\title{
SISTEMA DE CONTROLE E SUPERVISÃO PARA A PLANTA DE BENEFICIAMENTO DE MINÉRIO DE FERRO - ITM SAMAMBAIA*
}

Ailton Lopes Junio ${ }^{1}$

\section{Resumo}

O projeto desenvolvido na ITM Samambaia - Itatiaiuçu - MG, de propriedade da Mineração Usiminas, teve como objetivo a implantação de um Sistema de Controle e Supervisão através do fornecimento de todo o equipamento de automação e desenvolvimento dos aplicativos de controle e supervisão. Este projeto torna possível a operação remota de toda a planta, através de uma sala de controle central, bem como, a automatização de processos como sequenciamento de partida e parada, e o monitoramento do funcionamento de todos os equipamentos da planta, além de ferramenta de acesso remoto via internet para monitoração e comando da planta, mediante política de segurança de acesso.

Palavras-chave:Mineração; Automação; Processo; Controle.

\section{CONTROLAND SUPERVISION SYSTEM FOR PROCESSING PLANT IRON ORE - ITM SAMAMBAIA}

\begin{abstract}
The project developed in Samambaia Mine - Itatiaiuçu - MG, owned by Usiminas Mining, aimed at the implementation of a System Control and Supervision by providing all equipment automation and development of control and supervision applications. This project makes it possible for remote operation of the entire plant in a control room, as well as the automation of processes such as sequencing starting and stopping, and monitoring the operation of all plant equipment, remote web access to monitor and control the plant through access security policy.

Keywords: Mining; Automation; Process; Control.
\end{abstract}

1 Técnico em Automação, Técnico do Departamento de Engenharia e Automação, TSA Tecnologia em Sistemas de Automação S.A., Belo Horizonte, MG, Brasil.

* Contribuição técnica ao 18Seminário de Automação e TI Industrial, 23 a 26 de setembro de 2014, São Paulo, SP, Brasil. 


\section{INTRODUÇÃO}

Para o projeto ITM Friáveis da Usiminas Mineração a TSA forneceu todos os equipamentos de automação, desde redes de campo como a Profinet até níveis de supervisão e gerenciamento. Para tanto foi necessário um estudo junto ao cliente e fornecedor de automação (Siemens) da melhor arquitetura a ser usada, respeitando a segurança da rede e flexibilidade de futuros upgrades.

O desenvolvimento de típicos de programação foi baseado em norma ISA para equipamentos e subequipamentos, obedecendo à hierarquia de planta conforme as áreas do processo.

Os típicos e intertravamentos do processo foram testados em bancada, tentando reproduzir ao máximo as condições encontradas no campo.

O Comissionamento foi realizado na planta da ITM Samambaia, em Itatiaiuçu - MG.

\subsection{Rede de Automação}

A arquitetura de rede foi baseada em Protocolos de comunicação específicos para cada função do processo. Abaixo um exemplo de configuração de redes baseada na arquitetura da família SIMATIC PCS7:

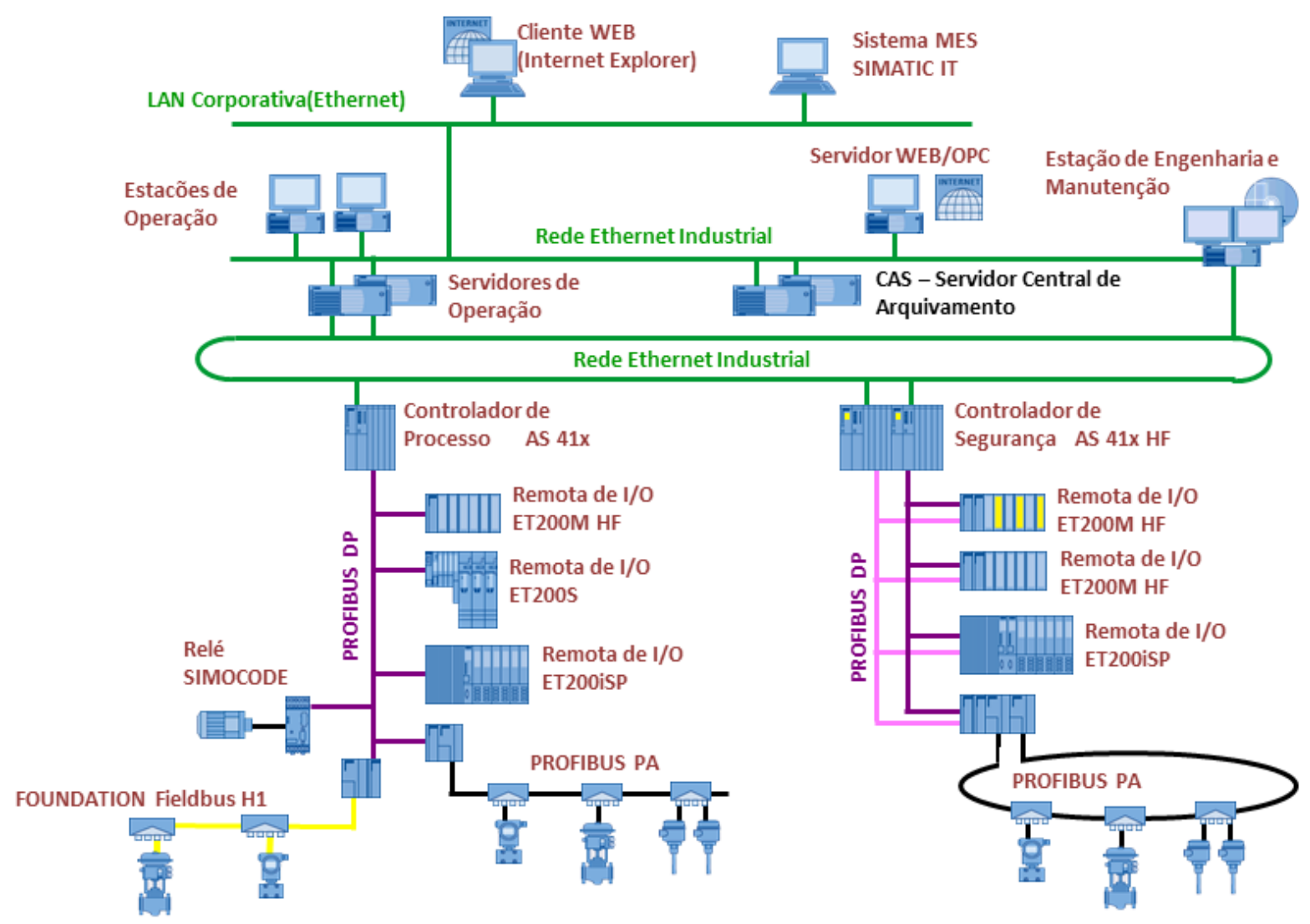

Figura 1 - Arquitetura SIMATIC PCS7.

ETHERNET: Ethernet é uma arquitetura de interconexão para redes locais - Rede de Área Local (LAN) - baseada no envio de pacotes. Ela define cabeamento e sinais elétricos para a camada física, e formato de pacotes e protocolos para a subcamada de controle de acesso ao meio (Media Access Control - MAC) do modelo OSI.1

\footnotetext{
* Contribuição técnica ao $18^{\circ}$ Seminário de Automação e TI Industrial, 23 a 26 de setembro de 2014,
} São Paulo, SP, Brasil. 


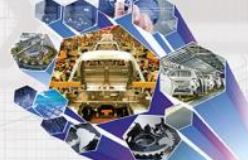

A Ethernet foi padronizada pelo IEEE como 802.3. Utilizamos dois níveis de redes para controle de tráfego e separação de níveis de acesso, mantendo a rede dos controladores livre de interferências da rede de supervisão.

PROFINET: PROFINET é uma rede baseada em um padrão de comunicação Ethernet Industrial padronizado pelas normas IEC 61158-5 e IEC 61158-6, 100\% compatível com a tecnologiaEthernet (IEEE 802.3) adotada pela associação $P I$ PROFIBUS \& PROFINET International.

PROFIBUS: PROFIBUS-DP (Decentralized Peripherals) Protocolo baseado em meio físico 485 Indicado para o chão de fábrica, onde há um grande volume de informações e necessidade de altas velocidades de comunicação para que os eventos sejam tratados em tempo adequado.

Para o projeto ITM Samambaia, utilizamos as redes citadas para as seguintes funções:

ETHERNET: Dois níveis de rede de dados.

Rede de Supervisão (TerminalBus). Rede para Estações de operação, Servidor de Acesso Remoto (WebServer), Historiador de Processo (PIMS) e Servidor do Sistema de Supervisão (WinCC).

Rede de Controle (PlantBus). Rede para Controladores de Campo (Automation Station), Estação de Engeharia e Servidor do Sistema de Supervisão (WinCC).

PROFINET: Redes de interligação das remotas de campo. Interligação em topologia Anel.

PROFIBUS: Redes para os Centros de Controle de Motores - CCM.

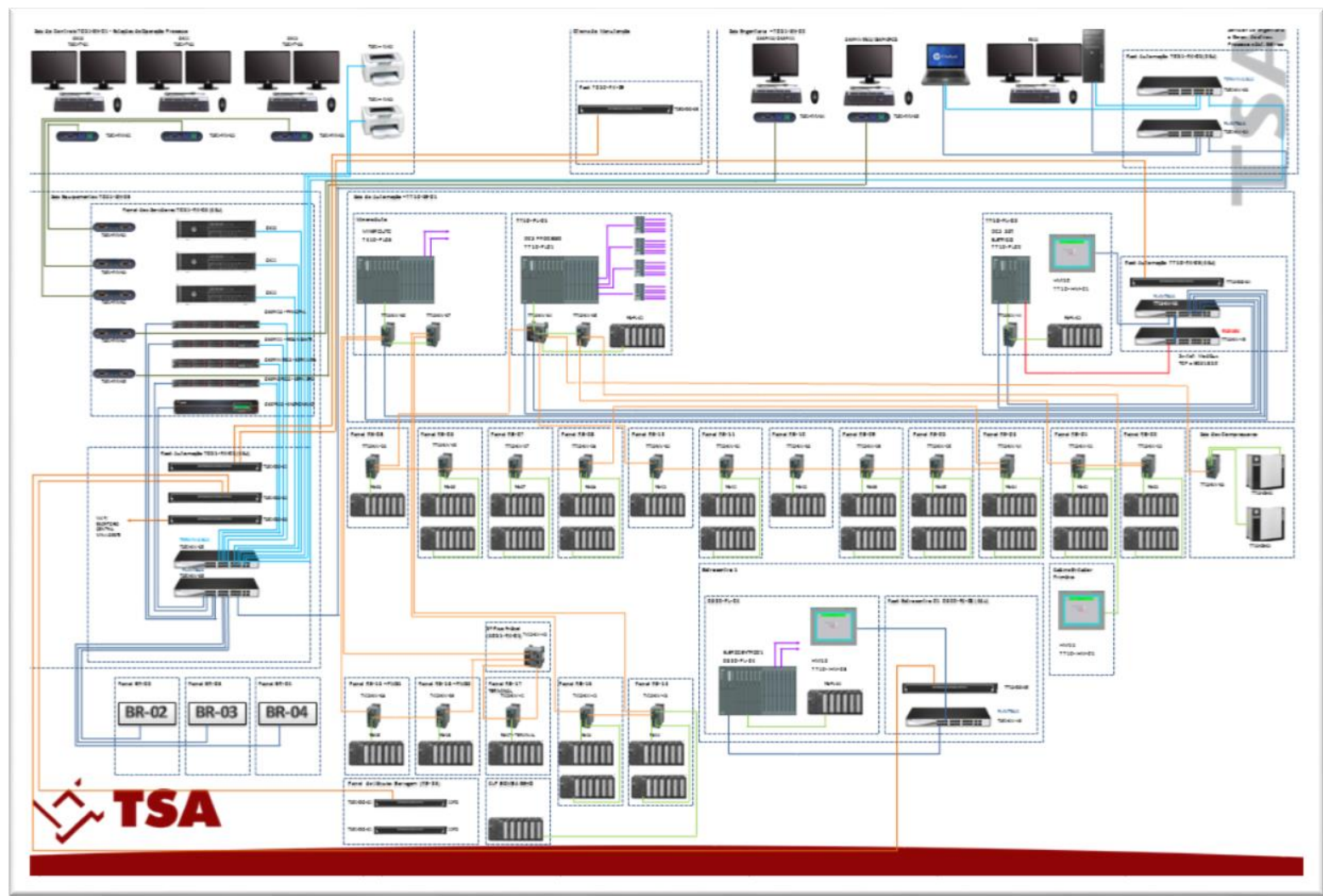

Figura 2 - Arquitetura simplificada da ITM Samambaia.

* Contribuição técnica ao $18^{\circ}$ Seminário de Automação e TI Industrial, 23 a 26 de setembro de 2014, São Paulo, SP, Brasil. 


\subsection{Estruturação de Áreas do Processo}

A divisão dentro do aplicativo do sistema de automação segue orientação da ISA, que divide o projeto em Site/Área/Sub-Área/Equipamento/Dispositivos. Estas divisões no PCS7 são chamadas de Hierarquia de Planta, sendo que as cartas lógicas bem como suas telas ou sequências de partida devem estar dentro do mesmo nível.

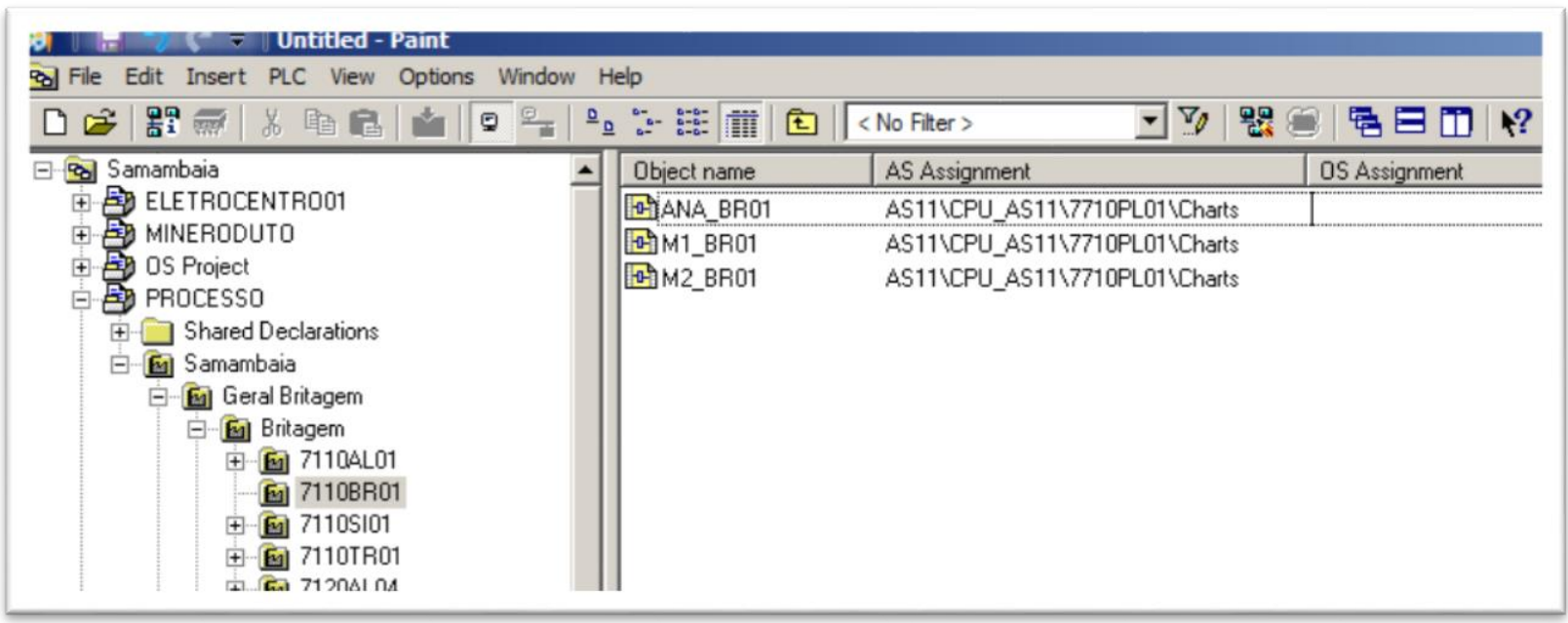

Figura 3 - Hierarquia de Planta.

\subsection{Configuração Gráfica de Funções Contínuas (CFCs) - Chart's}

Chart's são ambientes de programação orientados a blocos de funções onde desenvolvemos os típicos de equipamentos bem como seus automatismos, intertravamentos e proteções.

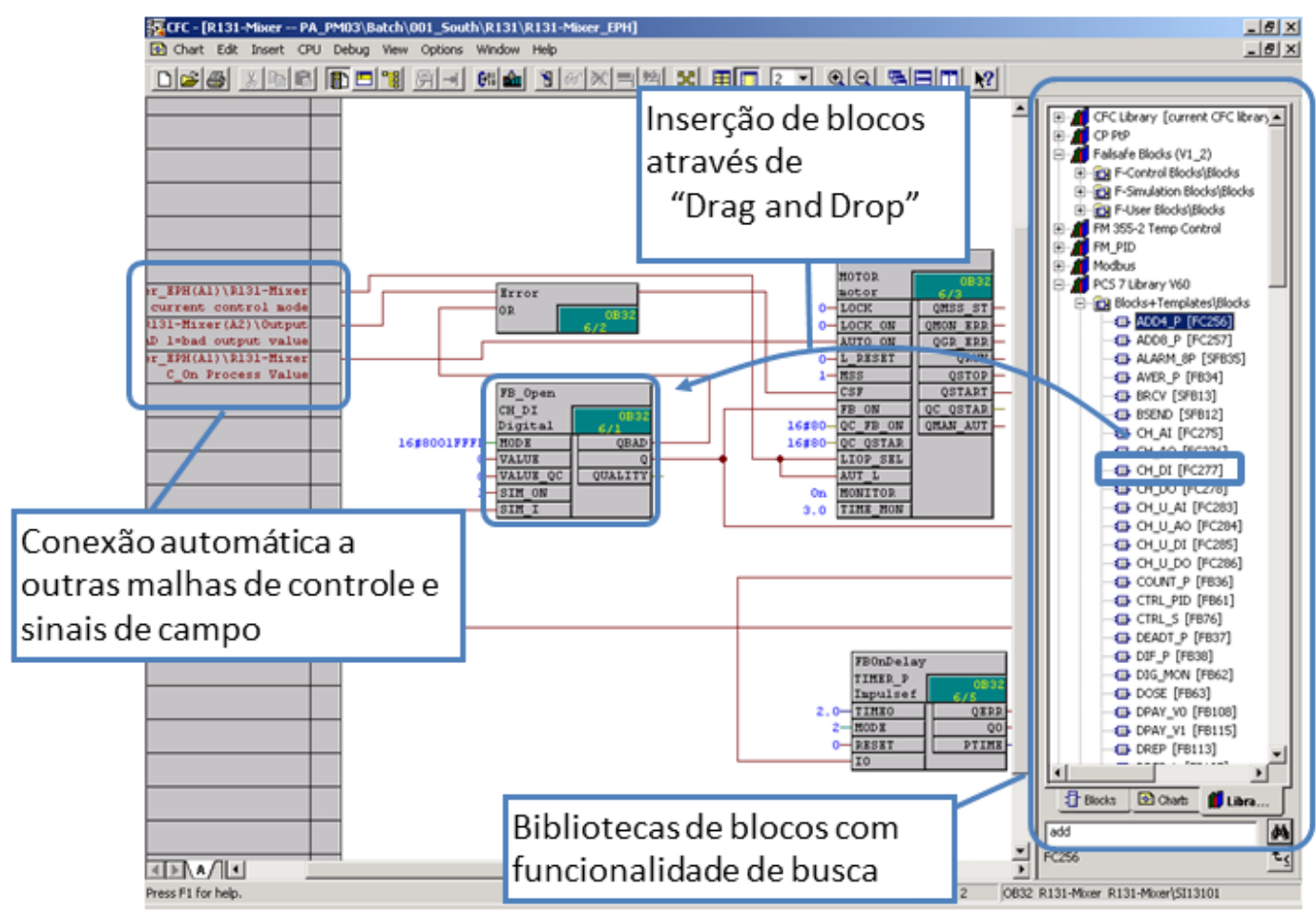

Figura 4 - Ambiente de programação - Chart's.

* Contribuição técnica ao $18^{\circ}$ Seminário de Automação e TI Industrial, 23 a 26 de setembro de 2014, São Paulo, SP, Brasil. 


\subsection{Controle Sequencial de Funções (SFC's)}

O controle sequencial de funções (SFC) é um sistema de controle executado em etapas, que, dependendo das condições, os avanços se dão de um estado para o outro.

Um sistema de controle seqüencial controla funções, como de CFC, através do modo e as mudanças de estado, e os processa de forma seletiva.

O SFC é utilizado para partidas e paradas de equipamentos. O conceito principal de funcionamento do SFC é o Passo Transição. A transição para o próximo passo somente acontece quando as condições solicitadas são satisfeitas, garantindo assim que não ocorra erros na sequência. Caso não haja retorno desta condição a sequência fica aguardando a condição ou o avnço manual do operador.

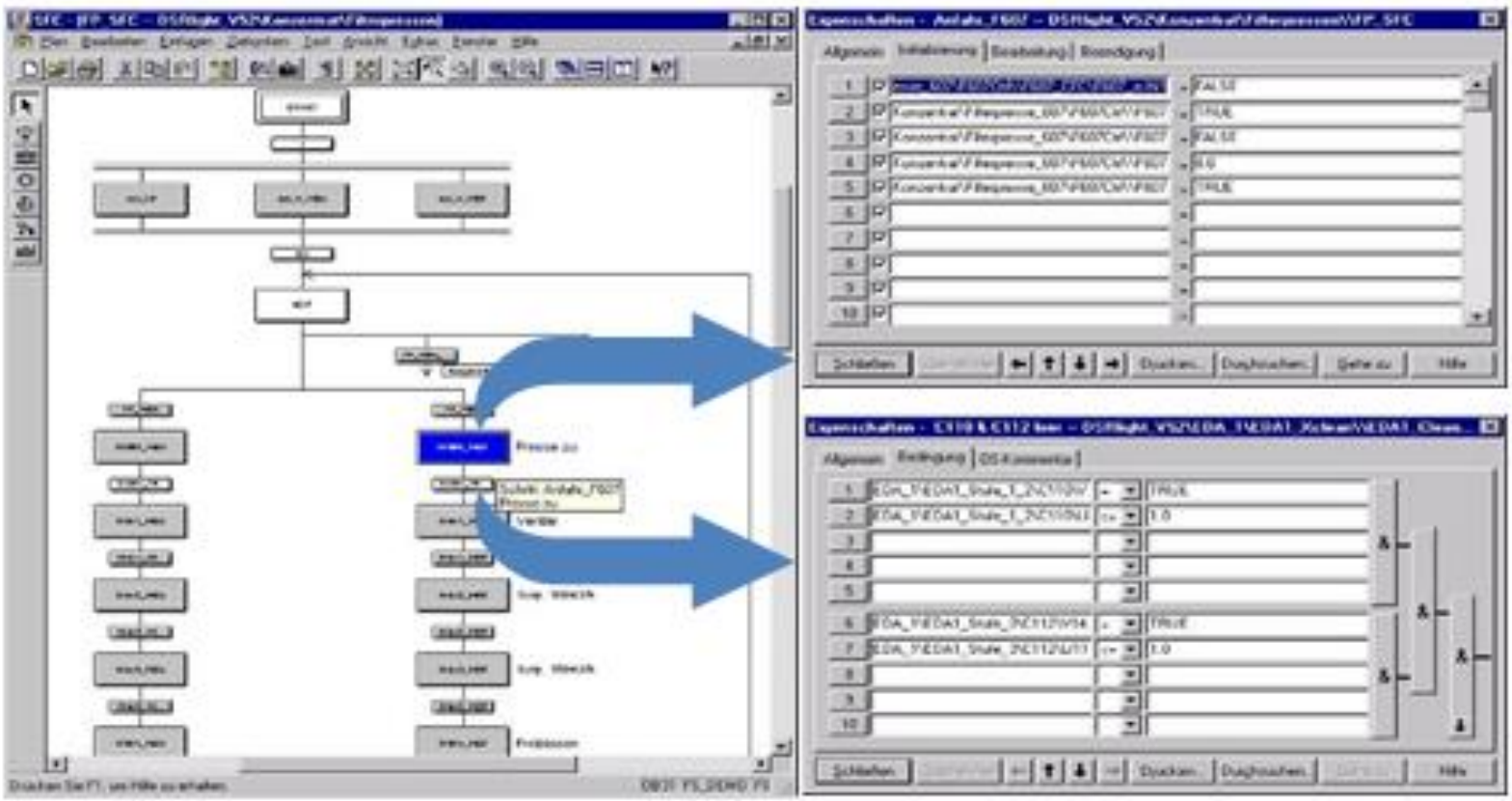

Figura 5 - Ambiente de programação de Sequenciamento

\subsection{Telas Gráficas, Block Icons e Faceplates}

As telas gráficas foram criadas reproduzindo ao máximo o fluxograma de processo, levando em consideração a ergonomia na utilização das mesmas pelo operador bem como a melhor representação cognitiva dos objetos, desconsiderando a escala de representação a fim de compreender todos os objetos de forma clara e inteligível no sinótico.

Os equipamentos e instrumentos possuem representação padrão por típico de equipamento. O PCS7 da Siemens possui uma ferramenta de criação automática de objetos baseado em bibliotecas, associados a equipamentos e instrumentos chamados de Block Icons. O Block Icon permite ao operador visualizar o estado do equipamento, bem como seus desvios e falhas. Outra função do Block Icon é chamar a janela de comando através do clicar do operador. As janelas de comandos são associadas ao típico do equipamento/Instrumento.

\subsubsection{Engenharia a partir da biblioteca de processo}

Exemplo: bloco de Interlock

\footnotetext{
* Contribuição técnica ao $18^{\circ}$ Seminário de Automação e TI Industrial, 23 a 26 de setembro de 2014,
} São Paulo, SP, Brasil. 


\begin{tabular}{|c|}
\hline Introdução \\
\hline $\begin{array}{l}\text { Componentes do } \\
\text { Sistema }\end{array}$ \\
\hline - Engenharia \\
\hline $\begin{array}{l}\text { - Interface de } \\
\text { Operaçã̃o }\end{array}$ \\
\hline $\begin{array}{l}\text { - Estaçãode } \\
\text { Manutenção }\end{array}$ \\
\hline - Periferia de Processo \\
\hline Integração com CCM \\
\hline Tecnologia \\
\hline Resumo \\
\hline
\end{tabular}

- Funcionalidades do bloco de Interlock:

- Indicação do estado no block icon (travado, destravado, by- passado, simulado, fora de uso)

- Visualização em cascata

- Salto direto para o faceplate

- Bypass / Indicação de "First Out"
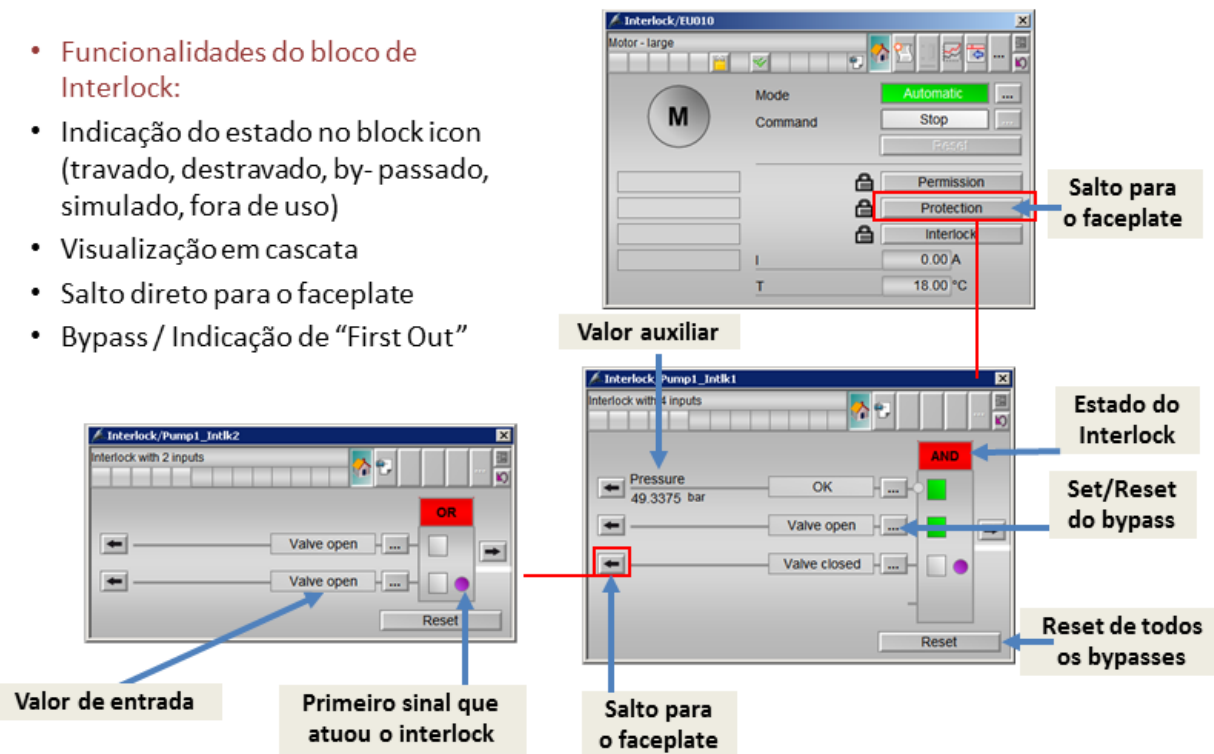

Grande variedade de funções prontas para utilização a partir das bibliotecas.

Figura 6 - Engenharia a partir de Bibliotecas

\subsection{Telas Gerais}

As telas gerais possuem uma representação geral do fluxo de processo de cada área da planta. Esta visão geral permite as chamadas das telas de processo, totalizadores e horímetros.

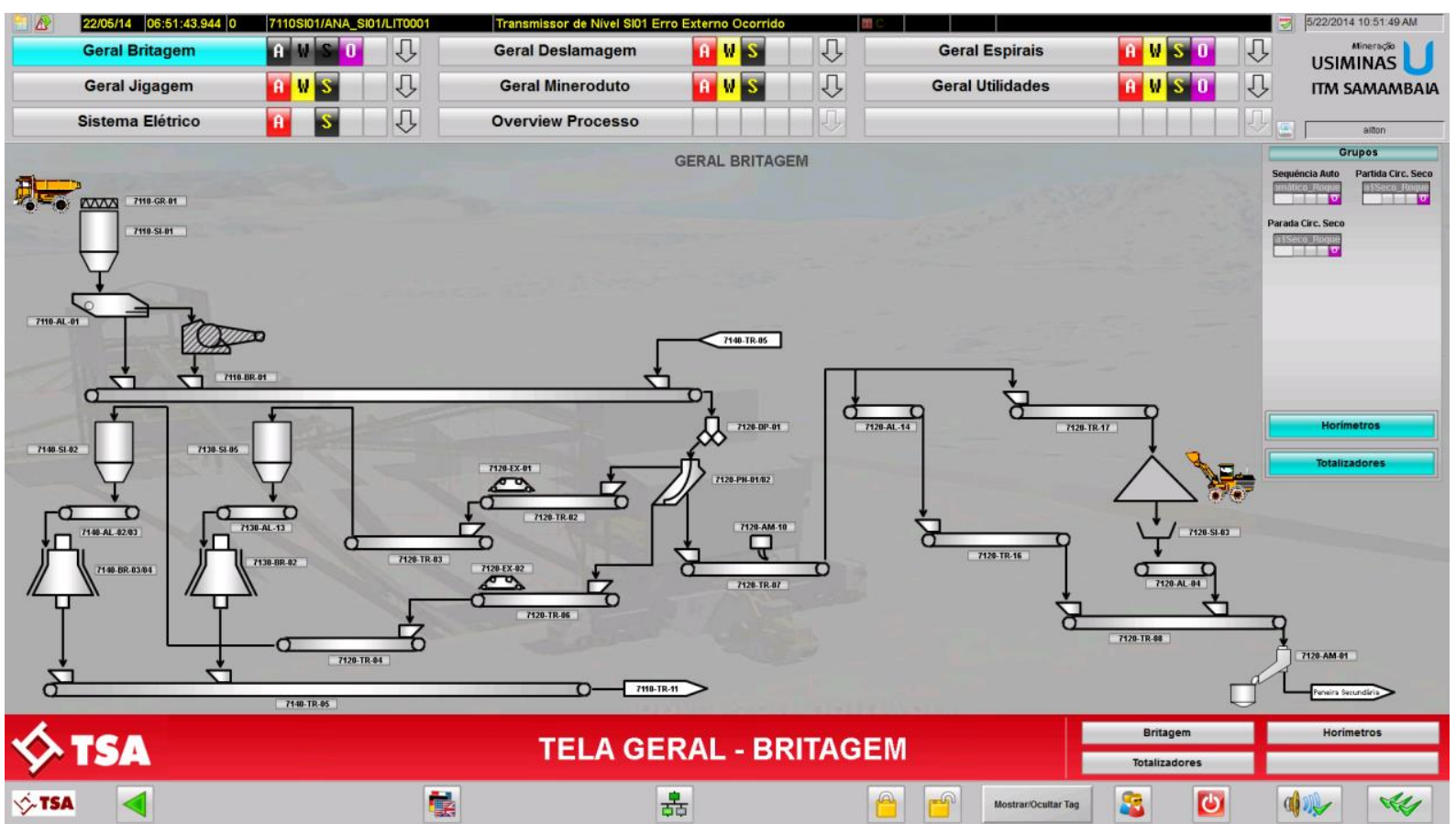

Figura 7 - Tela de Processo da Britagem.

\subsection{Telas de Processo}

As telas de processo permitem a visualização do estado atual do processo. Estas telas possuem Block Icons capazes de chamar as janelas de comandos de cada equipamento/Instrumento.

* Contribuição técnica ao 18Seminário de Automação e TI Industrial, 23 a 26 de setembro de 2014, São Paulo, SP, Brasil. 


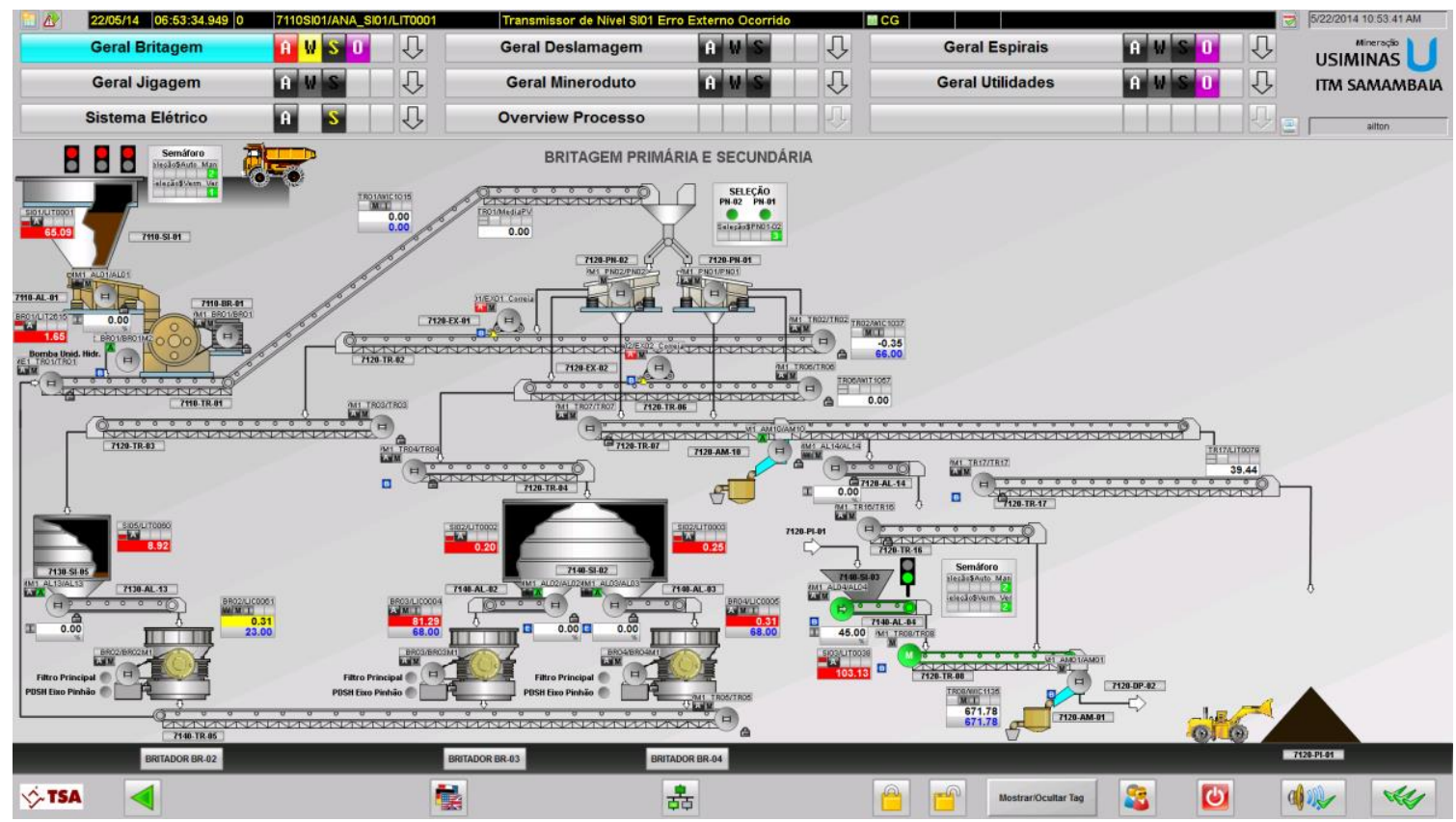

Figura 8 - Tela de Processo da Britagem.

\subsection{Sistema Integrado de gerenciamento de Alarmes}

A Interface do PCS7 permite uma clara interpretação dos eventos de desvio e parada de equipamento através de um sinótico de alarmes eficiente.

Esta ferramenta permite filtros, ordenações e tipificação de eventos, bem como apontar as ações dos usuários baseado na política de segurança e privilégios do WinCC.

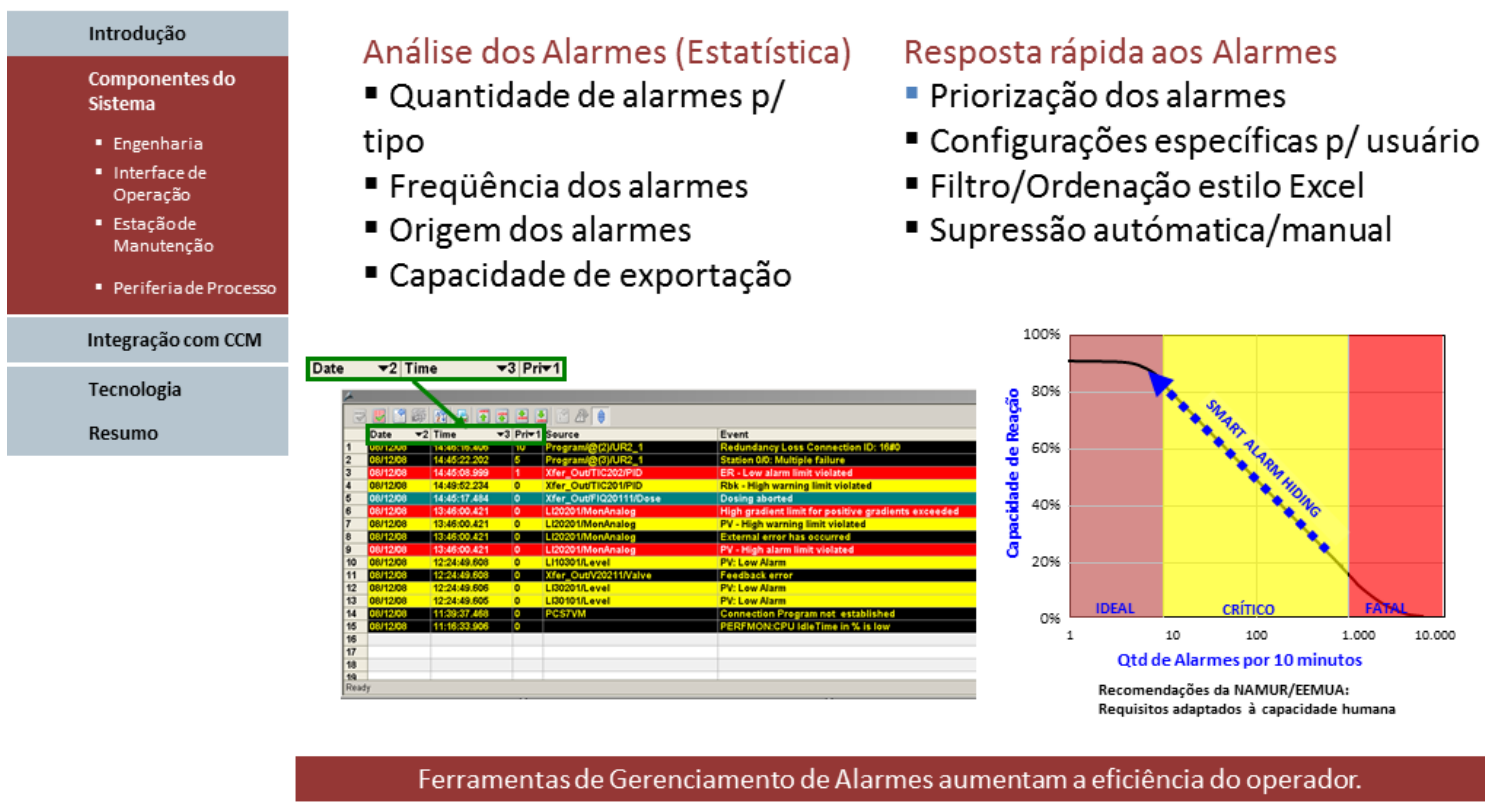

Figura 9 - Análise de Alarmes.

\section{MATERIAIS E MÉTODOS}

Para possibilitar a alta confiabilidade do sistema de controle foram utilizados Controladores Siemens da Linha 417, conectados entre si e aos diversos

\footnotetext{
* Contribuição técnica ao $18^{\circ}$ Seminário de Automação e TI Industrial, 23 a 26 de setembro de 2014,
} São Paulo, SP, Brasil. 


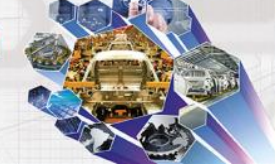

dispositivos do sistema através das redes Ethernet, PROFIBUS e PROFINET. O Sistema de Supervisão utilizou a última versão da plataforma PCS7 (Siemens).

Durante o desenvolvimento nas instalações da TSA em Belo Horizonte foram utilizados os típicos padrões da Siemens para não fugir do convencional. Entretanto, para cada tipo de IED - Dispositivo Eletrônico Inteligente foi necessário um estudo junto ao fabricante para prover a integração dos estados e diagnósticos ao Sistema de Supervisão e Controle PCS7.

Entre os principais dispositivos integrados ao sistema estão Relés de Sobrecarga em Estado Sólido para motores de partida direta e/ou com reversão (MM200/MM300-GE), inversores de frequência de baixa Tensão (VLT-Danfoss), inversores de frequência de média tensão (converteam-GE), relés de proteção de alimentadores e disjuntores de média e baixa tensão (F650/469-GE). (o alinhamento está diferente dos outros parágrafos)

Os diagnósticos dos IED's foram aquisitados de forma cíclica (polling), utilizando o Protocolo Profibus-DP DPV0. O tempo médio de varredura das redes que interligaram os CCM's da ITM Samambaia foi ajustado para $125 \mathrm{~ms}$.

Para a montagem das redes Profibus dos Centros de Controle de Motores - CCM utilizamos (escreva na terceira pessoa: foi utilizado) os critérios da seguinte literatura:

"PROFIBUS Guia de Instalação e Montagem de Cabos" - Ref 1, da Associação Profibus www.profibus.org.br.

Após a montagem das redes Profibus, bem com sua supervisão de montagem, a TSA realizou a análise e certificação das redes de comunicação.

Após a montagem das redes Profibus bem com sua revisão de montagem, foi feito um processo de analise e certificação das redes pela TSA.

A TSA possui as mais modernas ferramentas de análise de redes de comunicação do mercado, que possibilitam um diagnóstico rápido e preciso. O Profitrace 2 é a mais completa e poderosa ferramenta para análise e diagnóstico de redes PROFIBUS DP e PA disponível no mercado. O Profitrace 2 representa a última geração em tecnologia de monitoração de redes on-line porque combina em um só produto as funções de Analisador, Osciloscópio, Bargraph, Scan de Topologia e Master P-V0/V1.

\section{Bar Graph e Análise de Forma de Onda}

\section{a) Rede Cartão Profibus 1/ProfiHub 1 - Segmento 1 - Nós 3 a 12.}

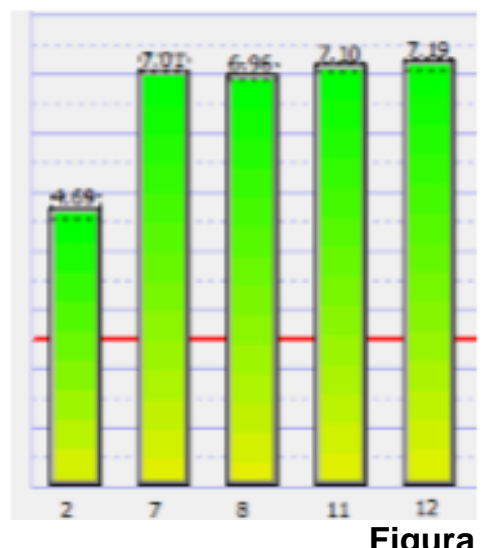

Figura 10 - Bar Graph - Nível de Sinal normal em todos os dispositivos

\footnotetext{
* Contribuição técnica ao $18^{\circ}$ Seminário de Automação e TI Industrial, 23 a 26 de setembro de 2014, São Paulo, SP, Brasil.
} 


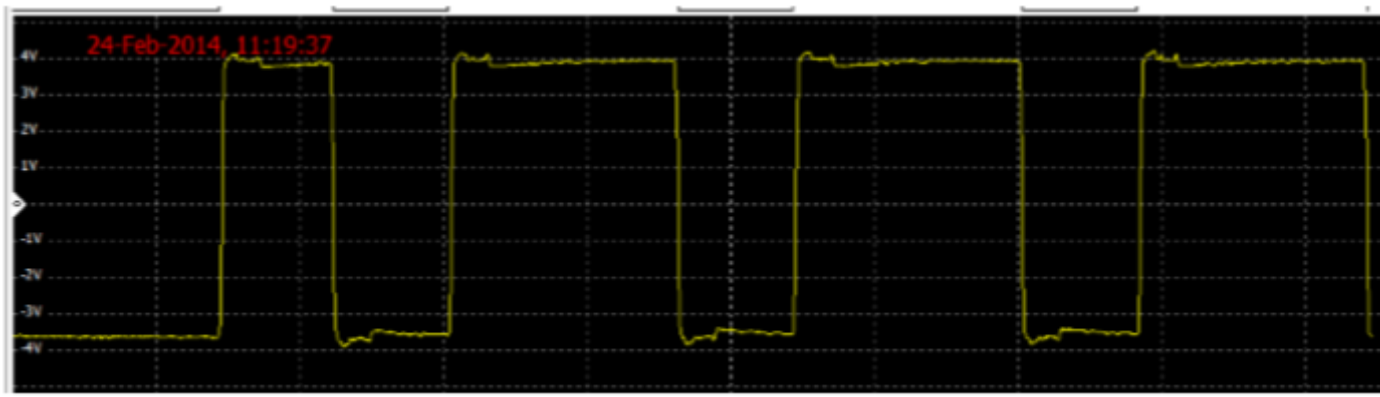

Figura 11 - Analise de forma de onda padrão para o segmento

\section{Considerações:}
$\checkmark \quad$ Nível do sinal pico a pico: Normal
$\checkmark$ Forma de onda: duração das bordas de subida e descida se encontram em seu valor ideal $0 / 16$, ou seja, praticamente instantâneo.
$\checkmark$ Tensão de Alimentação: Normal
$\checkmark$ Não foram detectados ruídos com a planta em funcionamento.

\section{RESULTADOS E DISCUSSÃO}

Os resultados obtidos com a automação da ITM Samambaia vão desde o controle integrado da planta, garantindo maior uniformidade do produto, até o controle e registro de variáveis, refletindo positivamente na produtividade da instalação.

Todos os equipamentos da Planta, incluído britadores, peneiras, correias transportadoras, jigues, espirais, bombas, hidro ciclones, espessadores, mineroduto, amostadores possuem comando centralizado e integrado em um único ponto. Isso aumenta a eficiência do controle e garante uma capacidade de resposta mais rápida na correção de desvios operacionais.

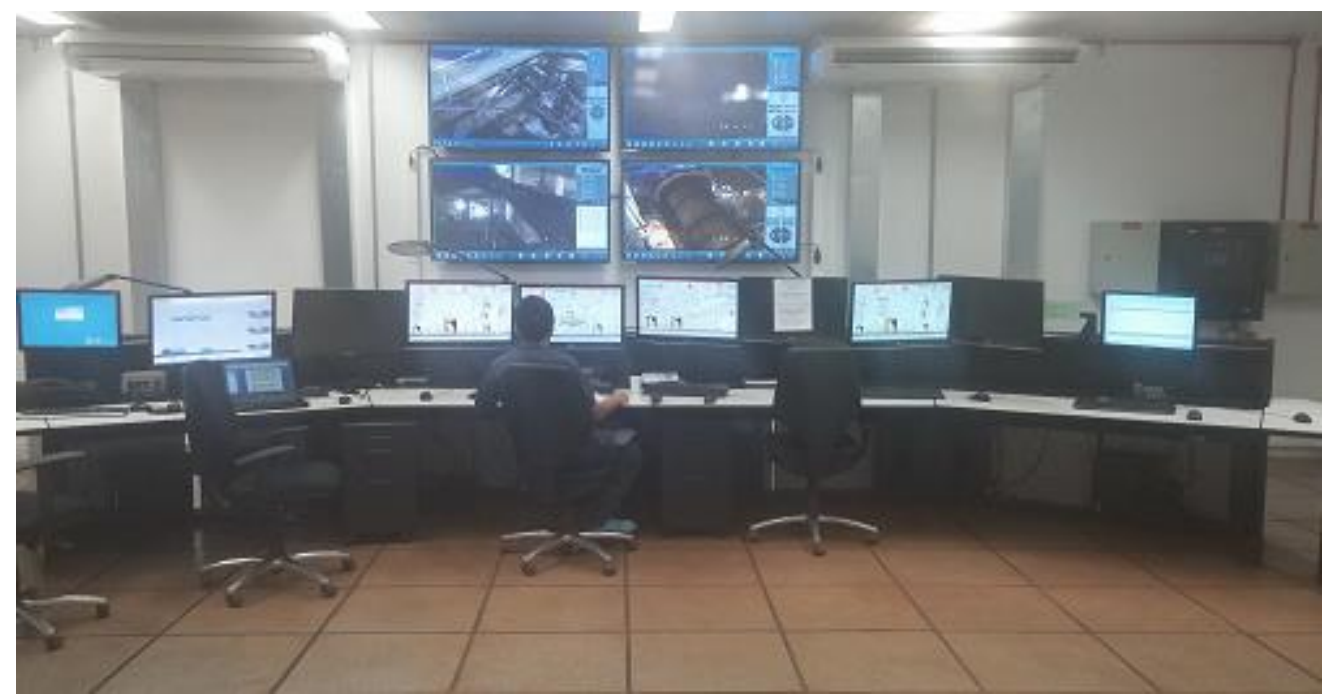

Figura 12 - Sala de Controle.

O diagnóstico do sistema PCS7 permite entender os problemas de hardware, redes, e equipamentos com um tempo de resposta rápido, sendo indispensável para a equipe de manutenção atuar ante os possíveis problemas físicos da planta.

A padronização das lógicas por típicos de configuração facilita a rápida interpretação da equipe de manutenção dos eventos de processo, defeitos de campo e

* Contribuição técnica ao 18Seminário de Automação e TI Industrial, 23 a 26 de setembro de 2014, São Paulo, SP, Brasil. 
intertravamentos de processo, auxiliando em rápidas correções momentâneas do processo.

\section{CONCLUSÃO}

O controle integrado da ITM Samambaia atua de forma constate e precisa na manutenção das variáveis de processo, buscando incessantemente o resultado pretendido. Os equipamentos trabalham sempre próximos à sua curva ótima de desempenho. Desta forma o resultado final da Planta é uma maior quantidade de produto entregue com um consumo mínimo de insumos como água, energia elétrica e reagentes químicos.

\section{Agradecimentos}

Agradecemos a Siemens Brasil, em especial aos senhores Valdex Sandro Gregório, que nos apoiou em todas as etapas do projeto. Agradecemos também ao senhor Aurélio Gentillini da GE Digital Energy, que nos deu todo o suporte necessário em relés para criarmos a interface com o Sistema.

\section{FONTES}

1 PROFIBUS Guia de Instalação e Montagem de Cabos

2 PROFIBUS Guide Order No: 8.022 Identification: Office-04-0007.

3 Arquitetura de Rede. Disponível em: www.siemensautomation.com

4 Aplicativo ITM Samambaia.

* Contribuição técnica ao $18^{\circ}$ Seminário de Automação e TI Industrial, 23 a 26 de setembro de 2014, São Paulo, SP, Brasil. 